\title{
Methanolic Extract of Plectranthus tenuiflorus Attenuates Quorum Sensing Mediated Virulence and Biofilm Formation in Pseudomonas aeruginosa PAO1
}

\author{
Sairengpuii Hnamte ${ }^{1}$, Pattnaik Subhaswaraj ${ }^{1}$, Sampath Kumar Ranganathan ${ }^{2}$, \\ Dinakara Rao Ampasala², Gangatharan Muralitharan ${ }^{3}$ and Busi Siddhardha ${ }^{1 *}$ \\ ${ }^{1}$ Department of Microbiology, School of Life Sciences, Pondicherry University, Puducherry - 605014 , India. \\ ${ }^{2}$ Centre for Bioinformatics, School of Life Sciences, Pondicherry University, Puducherry - 605 014, India. \\ ${ }^{3}$ Deparment of Microbiology, School of Life Sciences, Bharathidasan University, Tiruchirappali- 620024, India.
}

\begin{abstract}
Pseudomonas aeruginosa is an important opportunistic pathogen which causes bacterial keratitis, cyctic fibrosis and other hospital acquired infections. Its ability to form biofilm provides resistance to conventional antibiotics and further corroborates the global health concerns. The antibiotic resistance by $P$. aeruginosa is due to highly complex cellular signaling system called quorum sensing (QS). As QS controls bacterial pathogenicity and plays a crucial role in biofilm formation, it is a promising alternative target to combat the bacterial virulence. The present study aims to determine the inhibitory activity of Plectranthus tenuiflorus extract on QS and biofilm development in P. aeruginosa PAO1. The crude plant extract inhibited the production of pyocyanin, elastase, protease and chitinase by $71.96 \pm 1.82$, $38.74 \pm 1.29,30.84 \pm 1.20$ and $44.75 \pm 1.40 \%$ respectively at sub-MIC concentration of $500 \mu \mathrm{g} / \mathrm{ml}$. The production of biofilm aggravating phenotypes such as exopolysaccharides, alginate and rhamnolipid were also significantly reduced. The biofilm inhibition capability of $P$. tenuiflorus was further supported by light microscopic and confocal laser scanning microscopic analysis. The phytochemicals such as phytol and mosloflavone were identified from the crude extract using gas chromatography-mass spectrometry (GC-MS). The role of these phytochemicals in down regulation of QS in $P$. aeruginosa was further confirmed by in silico studies targeting transcriptional receptors, LasR and RhIR of the QS regulatory network. The in vitro and docking studies suggest the anti QS potential of $P$. tenuiflorus in combating the bacterial pathogenesis.
\end{abstract}

Keywords: Plectranthus tenuiflorus, Quorum sensing, Biofilm, GC-MS, Pseudomonas aeruginosa, Molecular docking.

*Correspondence: siddhardha.busi@gmail.com; +91-9597761788

(Received: 25 October 2018; accepted: 27 November 2018)

Citation: Sairengpuii Hnamte, Pattnaik Subhaswaraj, Sampath Kumar Ranganathan, Dinakara Rao Ampasala, Gangatharan Muralitharan and Busi Siddhardha, Methanolic Extract of Plectranthus Tenuiflorus Attenuates Quorum Sensing Mediated Virulence and Biofilm Formation in Pseudomonas aeruginosa PAO1, J Pure App/ Microbiol., 2018; 12(4):1985-1996. http://dx.doi. org/10.22207/JPAM.12.4.35

(c) The Author(s) 2018. Open Access. This article is distributed under the terms of the Creative Commons Attribution 4.0 International License which permits unrestricted use, sharing, distribution, and reproduction in any medium, provided you give appropriate credit to the original author(s) and the source, provide a link to the Creative Commons license, and indicate if changes were made. 


\section{INRODUCTION}

Quorum sensing (QS) is a cell density dependent signaling system in bacteria that supports the survival and virulence by coordinating the production of virulence factors ${ }^{1}$. Pseudomonas aeruginosa is a highly pathogenic bacterium causing pulmonary infections in the patients suffering from cystic fibrosis ${ }^{2}$. The chronic bacterial infections and associated health consequences by $P$. aeruginosa are due to highly evolved QS regulatory network. The QS phenomenon in $P$. aeruginosa comprises of highly recognized and intricately co-related cascade of four signaling systems such as LasI/R, Rhll/R, quinolone based PQS and integrated quorum sensing (IQS) systems ${ }^{3}$. Among the four interconnected QS hierarchy, the Las and Rhl system constitute two autoinducers synthase and their cognate receptors. The signaling molecules (autoinducers) generated from their respective AHL synthases bind with cognate receptors and control the expression of pyocyanin, exotoxins, elastolytic and proteolytic enzymes ${ }^{2}$. The QS regulatory network also involves in the formation, development and maturation of highly persistent biofilm matrix. The biofilm matrix is made of exopolysaccharides, rhamnolipids and alginates, which provide resistance to conventional antimicrobial therapy ${ }^{4}$. As QS mediates expression of virulence factors and controls development of biofilm, it provides novel platform for the development of anti-infective therapy.

In this context, from last few decades extensive research is being carried out in exploiting novel therapeutic agents targeting the bacterial QS and biofilm formation. Among the different strategies used to disrupt bacterial QS network, plant derived phytochemicals with proven biological activities represent an extensive class of quorum sensing inhibitors (QSIs) $)^{5}$. India represents one of the most important hotspots of medicinal plants with biodiversity. North-East India forms an important stretch of hotspot of medicinal plants with widespread distribution and rich variety of medicinal plants which have not been explored yet, thereby providing ample avenues for biomedical and pharmaceutical applications ${ }^{6}$. $P$. tenuiflorus is an important medicinal and ornamental shrub belongs to Lamiaceae family with ethnomedicinal importance. The folkloric use of $P$. tenuiflorus is well established as antiseptic, antioxidant, antimicrobial agent and for the treatment of nausea, abdominal disorders and respiratory infections ${ }^{7}$. In the present study, the anti QS and anti-biofilm activity of $P$. tenuiflorus was evaluated against $P$. aeruginosa $\mathrm{PAO} 1$ followed by docking studies.

\section{MATERIALS AND METHODS \\ Chemicals and reagents}

For determination of enzymatic activity, azocasein, elastin congo red (ECR) and chitin azure were purchased from Sigma Aldrich, USA. For other biological assays, chemicals like methanol, orcinol, crystal violet and acridine orange, reagents such as hydrochloric acid $(\mathrm{HCl})$, sulphuric acid $\left(\mathrm{H}_{2} \mathrm{SO}_{4}\right)$, calcium chloride $\left(\mathrm{CaCl}_{2}\right)$, Tris- $\mathrm{HCl}$ and dimethyl sulfoxide (DMSO) and the media Luria Bertani broth (LB broth), and LB agar were procured from HiMedia laboratories, India.

\section{Collection of Plants and Extract Preparation}

The leaf samples of $P$. tenuiflorus were collected from Manipur, India. The collected samples were cleaned and shade dried. The dried plant materials were grounded into fine powder. The powdered sample $(10 \mathrm{~g})$ was extracted with methanol $(100 \mathrm{ml})$ by continuous stirring for 48 h. After incubation, the solution was filtered, rotaevaporated and the obtained crude extract was stored for biological activities ${ }^{8}$.

\section{Maintenance of culture}

The anti QS activity of $P$. tenuiflorus was evaluated against biomarker strain, Chromobacterium violaceum (MTCC 2656) and test microrganism, P. aeruginosa PAO1 (MTCC 2453). The test cultures were obtained from IMTECH, Chandigarh, India.

\section{Screening of crude extracts for anti QS activity}

The preliminary anti QS activity of $P$. tenuiflorus crude extract was tested against $C$. violaceum (biomarker strain) and $P$. aeruginosa PAO1 using standard agar well diffusion method. Briefly, overnight bacterial culture was spread on top of the agar plates using sterile cotton swabs. Wells of $8 \mathrm{~mm}$ diameter were created and plant extract $(500 \mu \mathrm{g} / \mathrm{ml})$ was loaded. The zone of inhibition was measured after $24 \mathrm{~h}$ of incubation ${ }^{9}$. 


\section{Determination of Minimal inhibitory concentration (MIC)}

According to Clinical and Laboratory Standards Institute (CLSI, 2014), broth macrodilution method was used to determine the MIC of $P$. tenuiflorus extract against $P$. aeruginosa PAO1. All the biological activities were carried out at sub-MIC concentration ${ }^{10}$.

\section{Violacein inhibition assay}

Briefly, C. violaceum was incubated in presence of $P$. tenuiflorus extract at $30^{\circ} \mathrm{C}$ for $24 \mathrm{~h}$. After incubation, insoluble violacein was precipitated by centrifugation. DMSO was added to the pellet to solubilize the violacein pigment. The cell debris was removed by recentrifugation and the absorbance of violacein containing supernatant was determined at $585 \mathrm{~nm}^{11}$.

\section{Inhibition of QS controlled virulence in $P$.} aeruginosa PAO1

The production of pyocyanin, elastase, protease and chitinase are the important virulence factors which are regulated by QS. The inhibitory effect of $P$. tenuiflorus methanolic extract on the production of these virulence factors was evaluated. For pyocyanin inhibition, $P$. aeruginosa PAO1 was incubated in presence of $P$. tenuiflorus extract at $30^{\circ} \mathrm{C}$ for $18 \mathrm{~h}$. Pyocyanin pigment from cell-free supernatant was extracted using chloroform (5:3 ratio). The pyocyanin containing chloroform phase (blue organic phase) was reextracted with $0.2 \mathrm{M} \mathrm{HCl}$. The absorbance of the upper aqueous layer was determined at $520 \mathrm{~nm}^{12}$. The ablity of $P$. tenuiflorus extract on Staphylolytic activity of $P$. aeruginosa $\mathrm{PAO} 1$ was examined as per the standard protocol ${ }^{13}$.

For elastolytic activity, bacterial cell-free supernatant was mixed with ECR buffer $(100 \mathrm{mM}$ Tris, $1 \mathrm{mM} \mathrm{CaCl}, \mathrm{pH} \mathrm{7.5)} \mathrm{and} \mathrm{incubated} \mathrm{at} 37^{\circ} \mathrm{C}$ for $3 \mathrm{~h}$. After incubation, insoluble ECR was separated and the absorbance of the supernatant was measured at $495 \mathrm{~nm}^{14}$. For LasA protease activity, the cell free supernatant of $P$. tenuiflorus treated PAO1 was mixed with $0.3 \%$ azocasein $(50 \mathrm{mM}$ Tris- $\mathrm{HCl}, \mathrm{pH} 7.8$ ) and incubated for $30 \mathrm{~min}$. After incubation, undigested substrate was pelleted and the absorbance was determined at $400 \mathrm{~nm}^{15}$. For chitinase activity, $P$. aeruginosa $\mathrm{PAO} 1$ was added with sodium citrate buffer $(0.1 \mathrm{M}, \mathrm{pH} 4.8)$ containing chitin azure $(0.5 \mathrm{mg} / \mathrm{ml})$. The reaction mixture was incubated for 7 days with continuous stirring condition (100 rpm). After incubation, the undigested chitin azure was removed and absorbance was measured at $570 \mathrm{~nm}^{11}$.

\section{Inhibition of bacterial motility}

In $P$. aeruginosa PAO1, motility plays a crucial role during biofilm formation. The inhibitory potential of $P$. tenuiflorus extract on swimming and swarming motility was evaluated. Overnight grown $P$. aeruginosa PAO1 treated with plant extract was point inoculated into swimming medium (agar $0.3 \%$, tryptone $1 \%, \mathrm{NaCl} 0.5 \%$ ) and swarming medium $(0.5 \%$ agar, $0.5 \%$ filter sterilized glucose, $1 \%$ peptone, $0.5 \% \mathrm{NaCl}$ ) and incubated at $37^{\circ} \mathrm{C}^{16}$.

Inhibition of biofilm formation in P. aeruginosa PAO1

The process of biofilm formation and development is regulated by QS network. The biofilm is composed of carbohydrate residues like exopolysaccharides, rhamnolipids and alginate which are responsible for antibiotic resistance. The effect of methanolic extract on biofilm formation was evaluated. For biofilm inhibition, crystal violet assay was performed with slight modification. Overnight grown P. aeruginosa PAO1 was incubated in the freshly prepared media on treatment with plant extract for 16-18 h. After incubation, planktonic cells containing media were removed and the biofilm containing microtiter plate was washed with PBS to remove excess of planktonic cells. The biofilm cells were then stained with crystal violet $(0.4 \%)$ for $10 \mathrm{~min}$. After incubation, the crystal violet bound biofilms were dissolved in $95 \%$ ethanol and the optical density was measured at $540 \mathrm{~nm}^{16}$.

For EPS inhibition assay, P. tenuiflorus extract treated $P$. aeruginosa $\mathrm{PAO} 1$ was centrifuged and the resulting pellet was resuspended in high salt buffer. The solution was recentrifuged and to the supernatant, chilled ethanol was added and incubated overnight $\left(4^{\circ} \mathrm{C}\right)$. The extracted EPS was then quantified by standard phenol-sulphuric acid method ${ }^{17}$. The effect of $P$. tenuiflorus extract on alginate production was evaluated as per the standard carbazole method. In brief, $P$. aeruginosa PAO1 culture treated with $P$. tenuiflorus extract was mixed with boric acid-sulphuric acid solution. The reagent mixture was vortexed followed by addition 
of carbazole solution $(0.2 \%)$ and incubated at $55^{\circ} \mathrm{C}$ for $30 \mathrm{~min}$. After the incubation, optical density was measured at $530 \mathrm{~nm}^{18}$. For rhamnolipids inhibition, modified orcinol method was followed according to the method described by Banerjee et al. $(2017)^{19}$.

Cell surface hydrophobicity is crucial for bacterial adhesion during biofilm formation. The effect of $P$. tenuiflorus extract on bacterial CSH was evaluated by microbial adhesion to hydrocarbon (MATH) assay. Briefly, overnight culture of $P$. aeruginosa PAO1 cultivated in the presence of crude plant extract was centrifuged at 12,000 rpm for $5 \mathrm{~min}$. The pellet was washed three times with phosphate buffer and finally resuspended in ice-cold phosphate buffer. The absorbance of the resuspension was measured at $600 \mathrm{~nm}$ $\left(A_{0}\right)$. To the resuspended mixture, toluene (an aromatic hydrocarbon) was added and vigorously vortexed. After vortexing, the reaction mixture was allowed for phase separation and the absorbance of the aqueous phase was determined $\left(A_{1}\right)$ and hydrophobicity (\%) was calculated ${ }^{20}$.

Hydrophobicity $(\%)=\left[1-\left(A_{1} / A_{0}\right)\right] \times 100$

The effect of $P$. tenuiflorus extract on biofilm formation in $P$. aeruginosa PAO1 was evaluated by light and confocal laser scanning microscopic (CLSM) analysis. For microscopic studies, overnight $P$. aeruginosa PAO1 culture was inoculated into fresh growth medium containing cover glass of $1 \times 1 \mathrm{~cm}$ in 24-well Microtiter plate along with crude plant extract and incubated for 16 h. After incubation, the cover glasses were rinsed with distilled water to remove the planktonic cells. For light microscopy, the adhered biofilms on the cover glasses were stained with $0.4 \%$ crystal violet and then visualised under a light microscope at a magnification of 40x. Meanwhile, for CLSM analysis, acridine orange was used to stain the biofilm and visualised under CLSM (Model LSM710, Carl Zeiss, Jena, Germany) ${ }^{17}$.

Gas Chromatography-Mass Spectrometry (GCMS) analysis

The phytochemical profile of methanolic extract of $P$. tenuiflorus extract was identified by GC-MS analysis. The phytochemicals were separated in the column using Helium as carrier gas at a constant flow of $1 \mathrm{ml} / \mathrm{min}$. The phytochemicals present in the extract were identified based on the obtained spectrum and retention time using GC-MS NIST (2008) library.

\section{Molecular docking studies}

The docking studies were carried out in Schrodinger Maestro software v.11.5 (Schrodinger, LLC, New York, NY, 2018) and the binding affinity of identified phytochemicals from GC-MS analysis and natural autoinducers to transcriptional receptors, LasR and RhIR were analyzed. The ligand binding domain of LasR protein's 3D-strcture file (PDB ID: 2UV0) was retrieved from Protein Data Bank. As there is no experimental protein 3D-structure available for RhIR, protein sequence was retrieved from Uniprot database (ID: P54292.1) and the 3D-structures were predicted using ROBETTA web server. The obtained protein structures were validated using RAMPAGE web server (http:// mordred.bioc.cam.ac.uk/<"rapper/rampage. php) and the best model was selected for docking studies.

The LasR and RhIR proteins were subjected to preparation in protein preparation wizard of Maestro software v.11.5.Grid generation was performed in Glide, version 7.8 in Maestro software v.11.5, for LasR protein grid were defined around the active site residues (Tyr-56, Trp-60, Asp-73, Thr-75 and Ser-129) where autoinducer C12-homoserine lactone (C12-HSL) interacts with LasR protein whereas for RhIR, the grid was generated around the active site residue Trp- $68^{21}$, ${ }^{22}$.The above prepared grids were used for docking. The ligand compounds were obtained from PubChem database and submitted for preparation in LigPrep module in Maestro softwarev.11.5 and the prepared protein and ligand were subjected for docking. The 2D and 3D structures were generated using LigPlot+v.1.4.5 and Chimera v.1.6.2 respectively.

\section{Statistical Analysis}

All the experiments were performed in triplicates and the data was presented as mean \pm standard deviation (SD). For each assay, a control experiment (without the treatment of crude extract) was performed. All the obtained results were calculated as compared to the control. 


\section{RESULTS}

Screening of the plant extracts for anti QS activity

From the preliminary agar well diffusion assay, $P$. tenuiflorus extract exhibited zone of inhibition of 18 and $16 \mathrm{~mm}$ against biomarker strain, $C$. violaceum and test organism, $P$. aeruginosa $\mathrm{PAO} 1$ respectively at a concentration of $500 \mu \mathrm{g} / \mathrm{ml}$.

\section{Determination of MIC}

The MIC of $P$. tenuiflorus extract against $P$. aeruginosa PAO1 was found to be $750 \mu \mathrm{g} / \mathrm{ml}$ and $500 \mu \mathrm{g} / \mathrm{ml}$ was selected as the sub-MIC.

\section{Violacein inhibition assay}

On treatment with $P$. tenuiflorus extract, the production of violacein in C. violaceum was significantly reduced by $80.23 \pm 2.73 \%$ as compared to control.

Inhibition of QS regulated virulence factors in $\boldsymbol{P}$. aeruginosa PAO1

The pyocyanin production was significantly inhibited by $71.96 \pm 1.82 \%$ when treated with $P$. tenuiflorus extract (Figure 1). It also showed promising ability in inhibiting Staphylolytic activity of $P$. aeruginosa PAO1 by $18.21 \pm 2.01 \%$ (Figure 1). On treatment with plant extract, LasB elastase activity was inhibited by $38.74 \pm 1.29 \%$. A significant decrease in the Las A protease and chitinase activity was observed in $P$. aeruginosa PAO1 on treatment with $P$. tenuiflorus extract with an inhibition of $30.84 \pm 1.20$ and $44.75 \pm 1.40 \%$ respectively (Figure 1 ).

Inhibition of bacterial motility

On treatment with $P$. tenuiflorus extract, a significant decrease in swimming and swarming motility of $P$. aeruginosa was observed with a reduction of 37.93 and $54.54 \%$ respectively (Figure 2).

\section{Inhibition of biofilm formation in P. aeruginosa} PAO1

From the crystal violet based biofilm inhibition activity, it was observed that the biofilm formation was reduced by $44.03 \pm 2.00 \%$ on treatment with $P$. tenuiflorus extract. A significant reduction $(41.08 \pm 2.67 \%)$ in the production of EPS was observed in P. aeruginosa PAO1 when treated with $P$. tenuiflorus extract (Table 1). Meanwhile, on treatment with sub-MIC dose of $P$. tenuiflorus extract, alginate production in $P$. aeruginosa $\mathrm{PAO} 1$ was significantly inhibited by $37.13 \pm 3.93 \%$. The rhamnolipid production was also significantly altered on treatment with $P$. tenuiflorus extract with an inhibition of $34.95 \pm 2.49 \%$ as compared to untreated control. The treatment with the crude extract resulted in decrease of cell to cell surface attachment of $P$. aeruginosa PAO1 with a

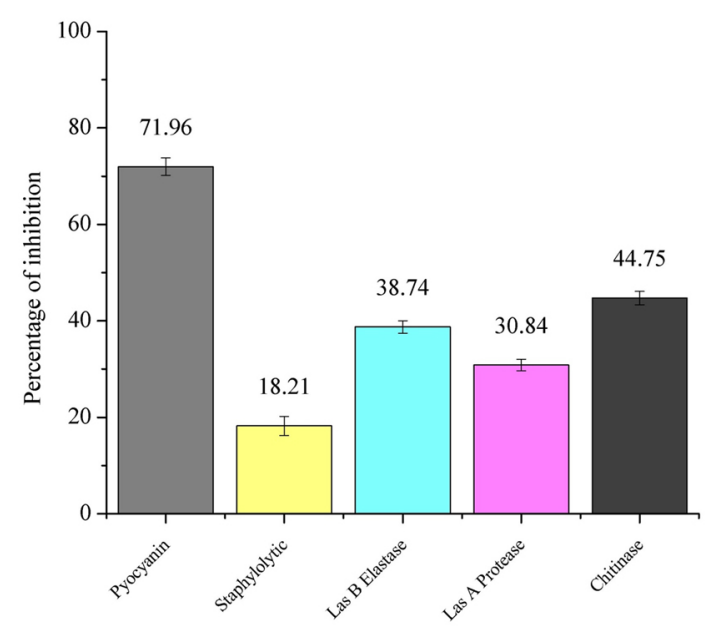

Fig. 1. Effect of sub-MIC dose $(500 \mu \mathrm{g} / \mathrm{ml})$ of $P$. tenuiflorus extract on production of $Q S$ regulated virulence factors such as pyocyanin, Staphylolytic activity, LasB elastase, LasA protease and chitinase activity

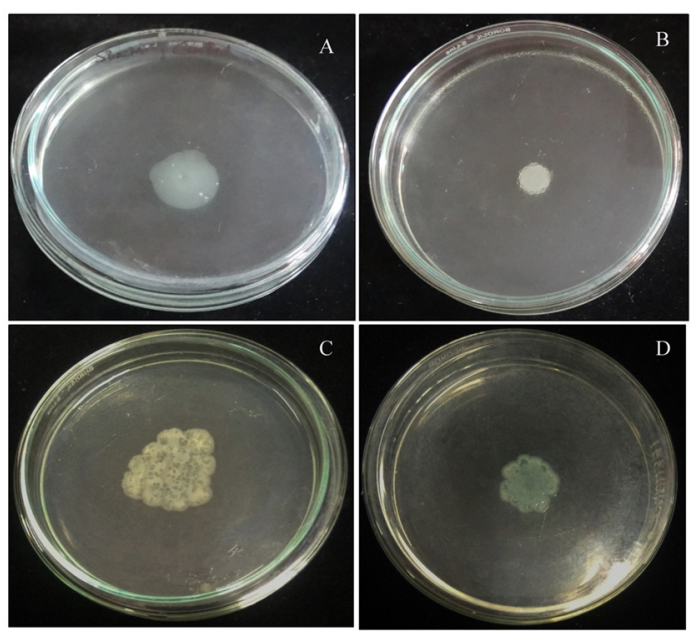

Fig. 2. Effect of sub-MIC dose $(500 \mu \mathrm{g} / \mathrm{ml})$ of $P$. tenuiflorus extract on the inhibition of bacterial motility (swimming and swarming) as compared to untreated control. (A) Swimming motility of untreated $P$. aeruginosa PAO1, (B) Swimming motility of $P$. tenuiflorus treated $P$. aeruginosa PAO1, (C) Swarming motility of untreated $P$. aeruginosa PAO1, (D) Swarming motility of $P$. tenuiflorus treated $P$. aeruginosa PAO1 

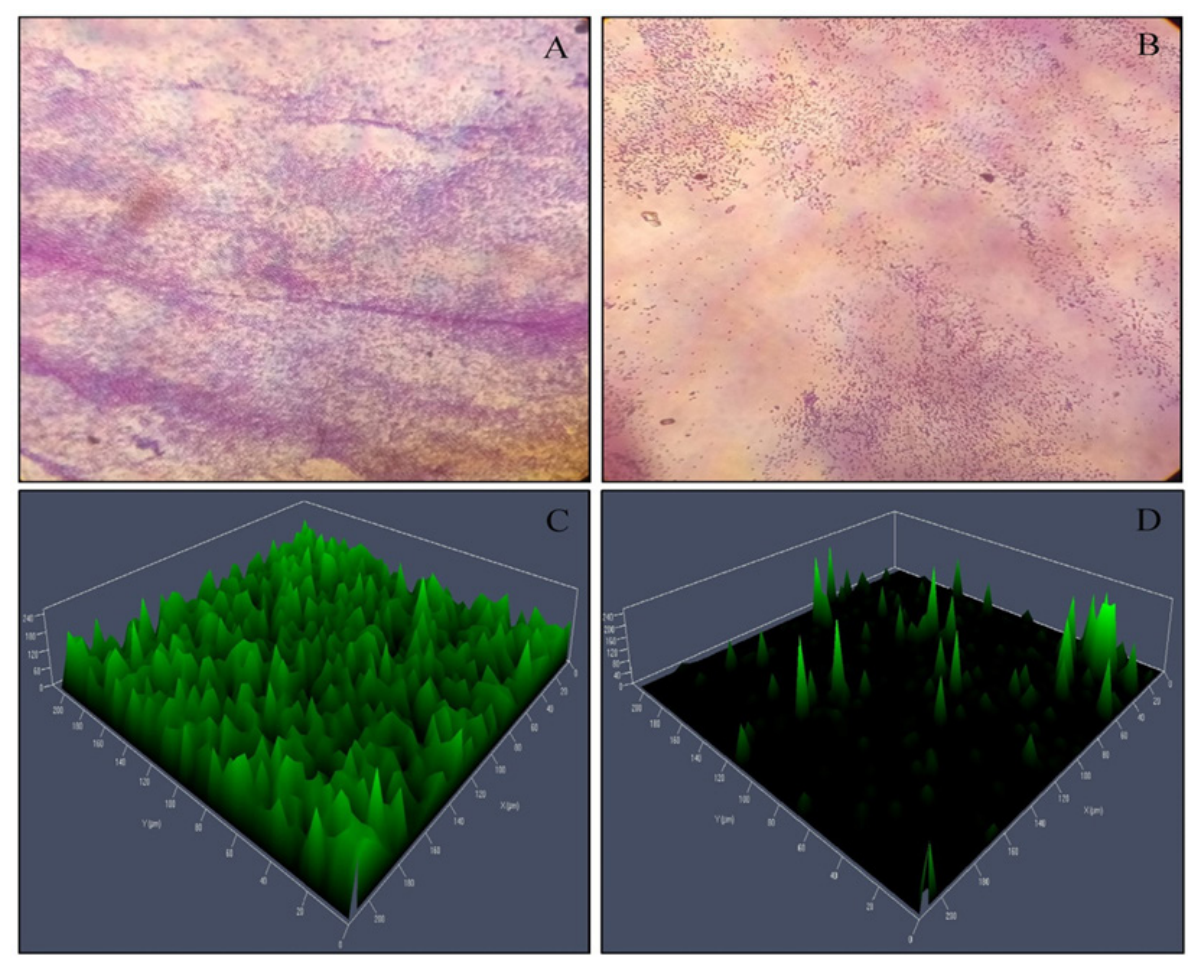

Fig. 3. Microscopic (Light microscopy and CLSM analysis) observation of $P$. aeruginosa PAO1 biofilm. (A) Light microscopic observation of untreated $P$. aeruginosa PAO1 biofilm, (B) Light microscopic observation of $P$. tenuiflorus treated $P$. aeruginosa PAO1 biofilm, (C) CLSM analysis of untreated $P$. aeruginosa PAO1 biofilm, (D) CLSM analysis of $P$. tenuiflorus treated $P$. aeruginosa PAO1 biofilm

Table 1. Effect of sub-MIC dose $(500 \mu \mathrm{g} / \mathrm{ml})$ of $P$. tenuiflorus extract on production of QS regulated biofilm phenotypes in $P$. aeruginosa PAO1

\begin{tabular}{lll}
\hline S. No. & QS regulated biofilm phenotypes & Inhibition (\%) \\
\hline 1. & Cell Surface Hydrophobicity & $29.19 \pm 3.29$ \\
2. & Rhamnolipid inhibition & $34.95 \pm 2.49$ \\
3. & Biofilm inhibition & $44.03 \pm 2.00$ \\
4. & EPS inhibition & $41.08 \pm 2.67$ \\
5. & Alginate inhibition & $37.13 \pm 3.93$ \\
\hline
\end{tabular}

Table 2. List of phytochemicals identified from methanolic extract of $P$. tenuiflorus using GC-MS analysis

\begin{tabular}{llcccc}
\hline $\begin{array}{l}\text { S. } \\
\text { No. }\end{array}$ & Phytochemicals & $\begin{array}{c}\text { Retention time } \\
(\mathrm{min})\end{array}$ & $\begin{array}{c}\text { Molecular } \\
\text { formula }\end{array}$ & $\begin{array}{c}\text { Molecular } \\
\text { weight }\end{array}$ & $\begin{array}{c}\text { Peak area } \\
(\%)\end{array}$ \\
\hline 1. & Beta.-D-glucopyranose, & & & & \\
& 1,6-anhydro & 13.463 & $\mathrm{C}_{6} \mathrm{H}_{10} \mathrm{O}_{5}$ & 162 & 3.212 \\
2. & N-Hexadecanoic acid & 18.400 & $\mathrm{C}_{16} \mathrm{H}_{32} \mathrm{O}$ & 256 & 23.731 \\
3. & Phytol & 19.765 & $\mathrm{C}_{20} \mathrm{H}_{40} \mathrm{O}$ & 296 & 4.324 \\
4. & Octadecanoic acid & 20.246 & $\mathrm{C}_{18} \mathrm{H}_{36} \mathrm{O}_{2}$ & 284 & 2.535 \\
5. & Mosloflavone & 25.433 & $\mathrm{C}_{17} \mathrm{H}_{14} \mathrm{O}_{5}$ & 298 & 12.526 \\
6. & Gamma sitosterol & 30.080 & $\mathrm{C}_{29} \mathrm{H}_{50} \mathrm{O}$ & 414 & 4.502 \\
\hline
\end{tabular}




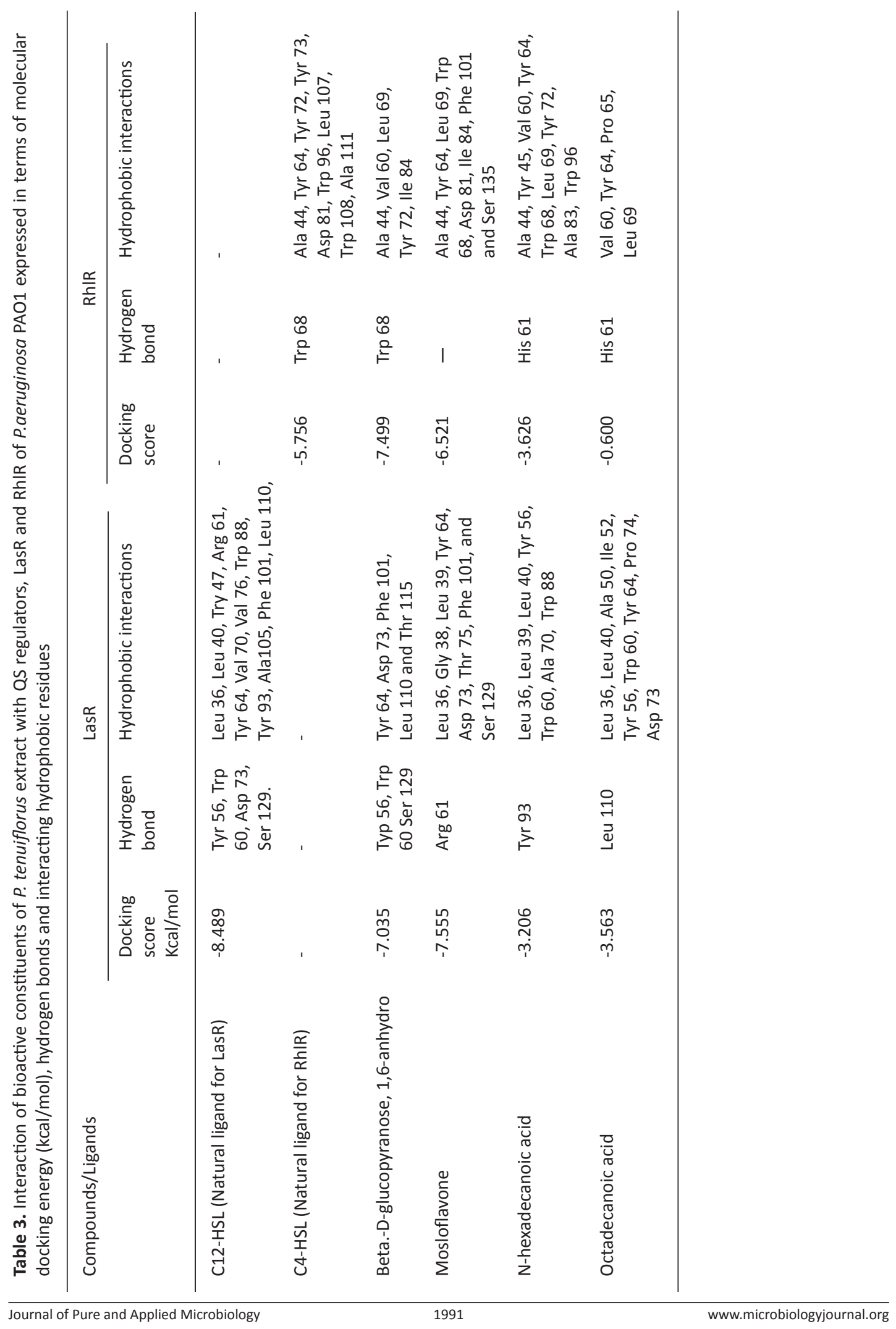


hydrophobicity percentage of $29.19 \pm 3.29 \%$ (Table 1). From light microscopic and CLSM analysis, a significant reduction in biofilm formation was observed in presence of $P$. tenuiflorus extract with dispersed bacterial cells, compared to thick and compact biofilm in untreated control (Figure 3).

\section{GC-MS analysis}

The phytochemical components identified from the methanol extract of $P$. tenuiflorus using GC-MS analysis were phytol, mosloflavone, N-hexadecanoic acid, Beta-D-glucopyranose, 1,6-anhydro and gamma sitosterol along with other minor phytoconstituents (Figure 4, Table 2).

\section{Molecular docking studies}

Molecular docking of $P$. teniflorus revealed that, mosloflavone exhibited a docking score of -7.55 and $-6.521 \mathrm{kcal} / \mathrm{mol}$ for LasR and RhIR respectively. The binding affinity of mosloflavone for RhIR was observed to be comparatively higher than its natural ligand suggesting the molecular target of mosloflavone (Table 3, Figure 5). Apart from mosloflavone, Beta-D-glucopyranose, 1,6-anhydro also showed greater affinity towards RhIR with a docking score of $-7.499 \mathrm{kcal} / \mathrm{mol}$ as compared to $-5.756 \mathrm{kcal} / \mathrm{mol}$ in case of natural ligand, C4-HSL (Figure 6).

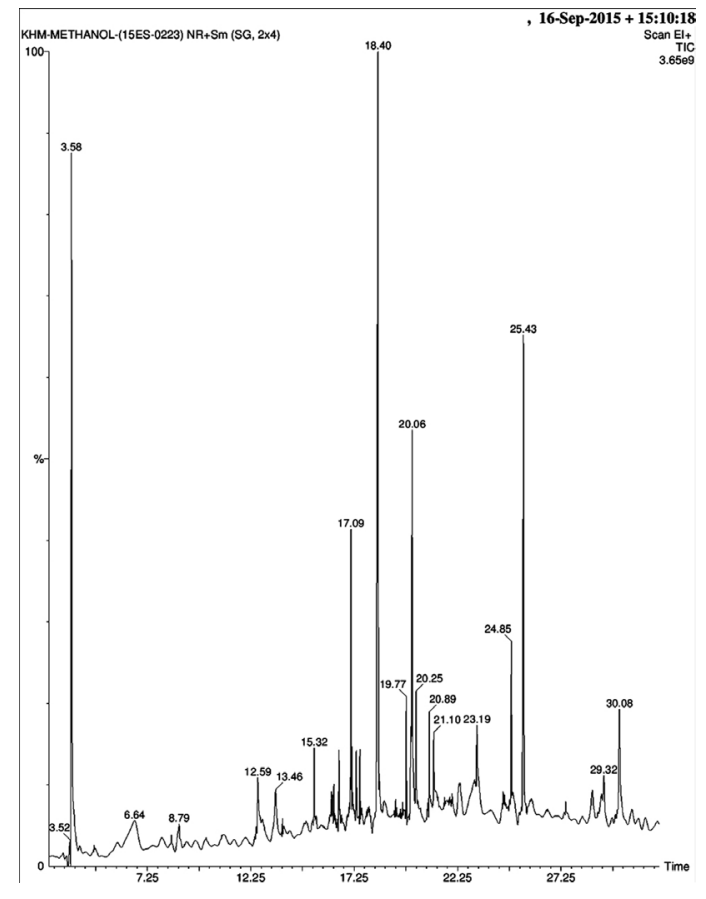

Fig. 4. GC-MS Chromatogram of methanolic extract of P. tenuiflorus

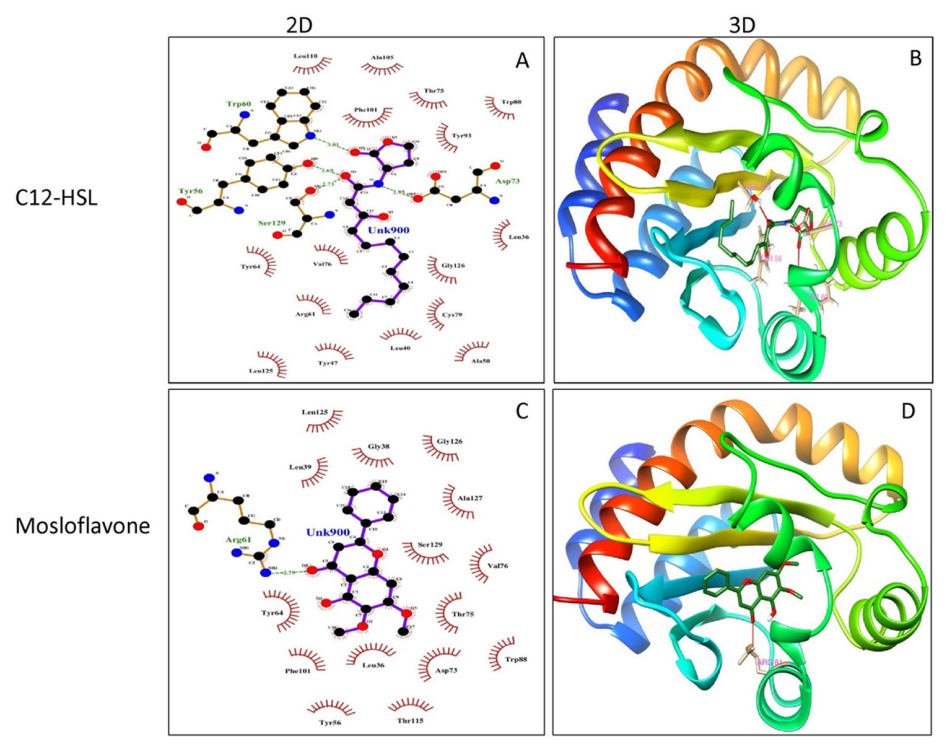

Fig. 5. Molecular docking studies of mosloflavone from crude extract of $P$. tenuiflorus to study the binding affinity with the active site of the receptor protein, LasR compared to the natural ligand (C12-HSL). (A) 2D docked conformation of C12-HSL (natural ligand) into the active site of LasR; (B) 3D docked conformation of C12-HSL into the active site of LasR, (C) 2D docked conformation of mosloflavone into the active site of LasR, (D) 3D docked conformation of mosloflavone into the active site of LasR. 


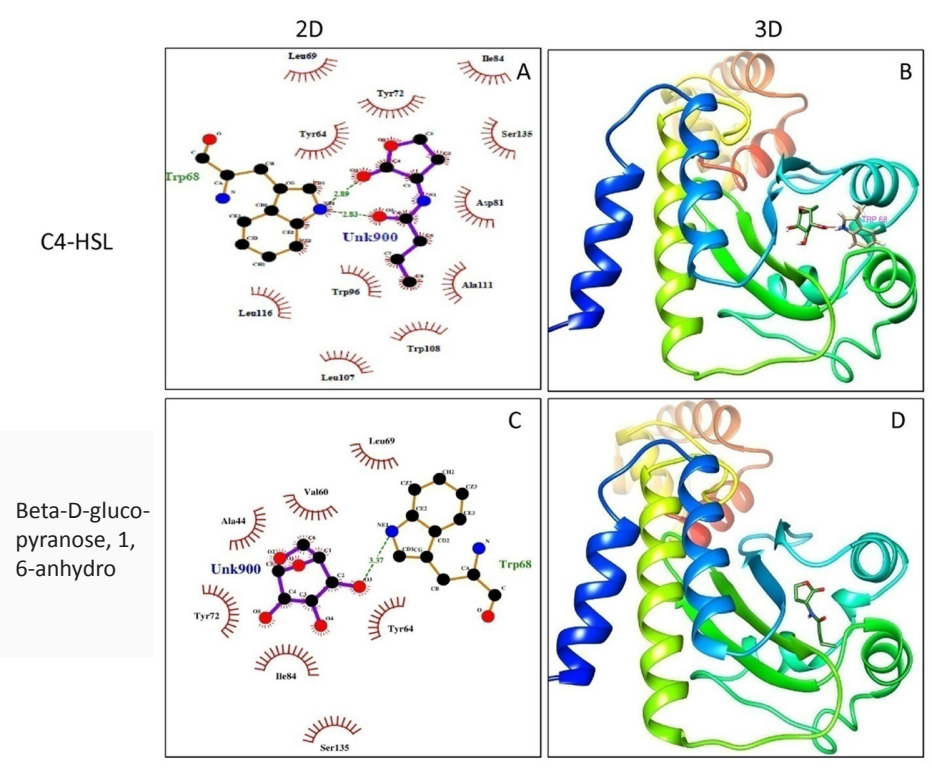

Fig. 6. Molecular docking studies of Beta-D-glucopyranose, 1, 6-anhydro from crude extract of $P$. tenuiflorus to study the binding affinity with the active site of the receptor protein, RhIR compared to the natural ligand (C4-HSL). (A) 2D docked conformation of C4-HSL (natural ligand) into the active site of RhIR; (B) 3D docked conformation of C4-HSL into the active site of RhIR, (C) 2D docked conformation of Beta-D-glucopyranose, 1,6-anhydro into the active site of RhIR, (D) 3D docked conformation of Beta-D-glucopyranose, 1,6-anhydro into the active site of RhIR.

\section{DISCUSSION}

Traditional medicinal plants are known for their ethnomedicinal value from several decades as the most affordable and easily accessible folkloric medicines for the treatment of various kinds of diseases including microbial infections ${ }^{23}$. India represents a rich heritage of medicinal plants with enormous ethnomedicinal properties of which majority of medicinal plants remain unnoticed and unexplored ${ }^{24}$. The methanolic extract of $P$. tenuiflorus was evaluated for its anti QS and anti-biofilm activity against $P$. aeruginosa PAO1. The plant extract significantly attenuated virulence factors production and biofilm formation regulated by QS regulatory network in $P$. aeruginosa with different potency. The production of violacein pigment, an important virulence factor of biomarker strain, $C$. violaceum is regulated by QS hierarchy. The methanolic extract of $P$. tenuiflorus significantly inhibited the production of violacein suggesting the efficacy in combating bacterial virulence ${ }^{25}$. Pyocyanin is an important member of the phenazine compounds produced by $P$. aeruginosa and represents a major virulence determinant by generating reactive oxygen species (ROS) ${ }^{26}$. In the present study, P. tenuiflorus inhibited the pyocyanin production by $71.96 \%$. The synergistic activity of phytochemicals like phytol and mosloflavone present in the crude extract could be responsible for pyocyanin inhibition ${ }^{27}$. The production of lytic enzymes such as LasA protease and LasB elastase by $P$. aeruginosa gives an aided advantage to the bacteria during host infection by enhancing the ability to degrade the host tissues ${ }^{28}$. In the present sudy, LasA protease and LasB elastase activity were significantly altered on treatment with $P$. tenuiflorus extract. These results suggested the ability of crude extract in imparing the ability of $P$. aeruginosa PAO1 to invade and degrade host tissues during infection. Chitinase is also an extracellular enzyme produced by $P$. aeruginosa and plays a crucial role during host invasion and survival within the host ${ }^{29}$. P. tenuiflorus extract significantly inhibited the chitinase activity of $P$. aeruginosa PAO1.

In addition to virulence factors production, the QS regulatory network also controls the formation, development and maturation of biofilm matrix, which is mainly responsible for increased 
antibiotic resistance ${ }^{30}$. The effect of crude extract on biofilm formation was quantified by crystal violet staining depicting the reduction in biofilm formation. This result was in accordance with the earlier report ${ }^{31}$. EPS and alginates not only provide assistance during bacterial adhesion but also shield the bacterial cells from oxidative stress, host immunity and antimicrobial treatment ${ }^{32}$. The production of EPS and alginate were inhibited when treated with $P$. tenuiflorus extract suggesting its role in disrupting the biofilm matrix. The production of biosurfactant, rhamnolipid plays a critical role in different stages of biofilm formation. In this context, combating rhamnolipid production could be a promising target for biofilm inhibition ${ }^{33}$. The rhamnolipid production was significantly altered on treatment with $P$. tenuiflorus extract suggesting its ability to inhibit biofilm formation. The bacterial motility plays an important role in the bacterial pathogenicity and biofilm formation. The swimming and swarming motility of $P$. aeruginosa PAO1 were significantly inhibited when treated with the plant extract suggesting its efficacy in interrupting the process of biofilm formation. These results were in accordance to the earlier report ${ }^{34}$. The in vitro anti-biofilm efficacy of crude plant extract was further corroborated by microscopic analysis depicting the reduction in the biofilm architecture as compared to compact biofilm architecture in the untreated control.

The presence of phytol in the methanolic extract of $P$. tenuiflorus, as identified from GC-MS analysis suggested the efficacy of crude extract in combating QS regulated virulence and biofilm formation in $P$. aeruginosa PAO1 ${ }^{27}$. The in vitro results were further validated using molecular docking studies of the identified phytochemicals for their affinity towards LasR and RhIR as compared to their respective autoinducers. Among the identified phytochemicals, mosloflavone and Beta.-D-glucopyranose, 1,6-anhydro exhibited promising affinity towards LasR and RhIR respectively suggesting their role in inhibiting bacterial QS system in P. aeruginosa PAO1.

\section{CONCLUSION}

The present study illustrated the importance of $P$. tenuiflorus as a potent QS inhibitor and biofilm inhibitor of $P$. aeruginosa
PAO1. The promising anti QS and anti biofilm activity exhibited by $P$. tenuiflorus extract was the result of synergistic activity of different phytochemicals such as phytol, mosloflavone and Beta.-D-glucopyranose, 1,6-anhydro present in the extract. The in vitro results were further validated by molecular docking studies depicting the binding ability of phytochemicals to LasR and RhIR of QS regulatory network. The present study will provide a lead in the antimicrobial drug discovery for the development of anti-infectives by targeting the QS regulated bacterial virulence and biofilm formation.

\section{CONFLICT OF INTEREST}

The authors declare no conflict of interest.

\section{ACKNOWLEDGEMENT}

The authors are thankful to Bharathidasan University, Tiruchirappalli for using the CLSM facility. The authors would also like to thank Sophisticated Instrumentation Facility, VIT University, VIT-SIF Lab, SAS, Chemistry Division for GC-MS Analysis.

\section{REFERENCES}

1. Schuster M, Sexton D J, Diggle S P, Greenberg E P. Acyl-homoserine lactone quorum sensing: From evolution to application. Annu Rev Microbiol, 2013; 67: 43-63.

2. Lee J, Wu J, Deng Y, Wang J, Wang C, Wang J, Chang C, Dong Y, Williams P, Zhang L H. A cellcell communication signal integrates quorum sensing and stress response. Nature Chem Biol, 2013; 9: 339-343.

3. Lee J, Zhang L. The hierarchy quorum sensing network in Pseudomonas aeruginosa. Protein Cell, 2015; 6(1): 26-41.

4. Brackman G, Coenye T. Quorum sensing inhibitors as anti-biofilm agents. Curr Phramaceut Des, 2015; 21: 5-11.

5. Bouyahya A, Dakka N, Et-Touys A, Abrini J, Bakri Y. Medicinal plant products targeting quorum sensing for combating bacterial infections. Asian Pacific J Trop Med, 2017; 10(8): 729-743.

6. Shankar R, Rawat M S. Conservation and cultivation of threatened and high valued medicinal plants in North East India. Int J Biodiv Conserv, 2013; 5(9): 584-591.

7. Waly N M, El Gayed S H. Botanical and biological studies of Plectranthus tenuiflorus (Vatke) Agnew. (Lamiaceae) growing in Saudi Arabia. 
Int J Life Sci Pharma Res, 2012; 2 (2): L52-L64.

8. Mostafa A A, Al-Askar A A, Almaary KS, Dawoud T M, Sholkamy E N, Bakri M M. Antimicrobial activity of some plant extracts against bacterial strains causing food poisoning diseases. Saudi J Biol Sci, 2018; 25: 361-366.

9. Burt S A, Ojo-Fakunle V T A, Woertman J, Veldhuizen E J A. The natural antimicrobial carvacrol inhibits quorum sensing in Chromobacterium violaceum and reduces bacterial biofilm formation at sub-lethal concentrations. PLOS ONE, 2014; 9(4): e93414.

10. El-Shaer S, Shaaban M, Barwa R, Hassan R. Control of quorum sensing and virulence factors of Pseudomonas aeruginosa using phenylalanine arginyl â-naphthylamide. J Med Microbiol, 2016; 65: 1194-1204.

11. Husain F M, Ahmad I, Al-thubiani A S, Abulreesh $\mathrm{H} \mathrm{H}$, AlHazza I M, Aqil F. Leaf extracts of Mangifera indica L. inhibit quorum sensing regulated production of virulence factors and biofilm in test bacteria. Front Microbiol, 2017; 8: 727.

12. Aybey A, Demirkan E. Inhibition of quorum sensing-controlled virulence factors in Pseudomonas aeruginosa by human serum paraoxonase. J Med Microbiol, 2016; 65: 105113.

13. Mohabi S, Kalantar-Neyestanaki D, Mansouri S. Inhibition of quorum sensing-controlled virulence factor production in Pseudomonas aeruginosa by Quercus infectoria gall extracts. Iran J Microbiol, 2017; 9(1): 26-32.

14. Vasavi H S, Arun A B, Rekha P D. Anti-quorum sensing activity of Psidium guajava $\mathrm{L}$. flavonoids against Chromobacterium violaceum and Pseudomonas aeruginosa PAO1. Microbiol Immunol, 2014; 58: 286-293.

15. Rajkumari J, Borkotoky S, Murali A, Suchiang K, Mohanty S K, Busi S. Attenuation of quorum sensing controlled virulence factors and biofilm formation in Pseudomonas aeruginosa by pentacyclic triterpenes, betulin and betulinic acid. Microb Pathog, 2018; 118: 48-60.

16. Pattnaik SS, Ranganthan S, Ampasala D R, Syed A, Ameen F, Busi S. Attenuation of quorum sensing regulated virulence and biofilm development in Pseudomonas aeruginosa PAO1 by Diaporthe phaseolorum SSP12. Microb Pathog, 2018; 118: 177-189.

17. Packiavathy I A S V, Priya S, Pandian S K, Veera Ravi A. Inhibition of biofilm development of uropathogens by curcumin - An anti-quorum sensing agent from Curcuma longa. Food Chem, 2014; 148: 453-460.
18. Gopu V, Meena C K, Shetty P H. Quercetin influences quorum sensing in food borne bacteria: in-vitro and in-silico evidence. PLOS ONE, 2015; 11(1): e0148471.

19. Lather P, Mohanty A K, Jha P, Garsa A K. Contribution of cell surface hydrophobicity in the resistance of Staphylococcus aureus against antimicrobial agents. Biochem Res Int, 2016; 2016: Article ID 1091290.

20. Banerjee M, Moulick S, Bhattacharya K K, Parai D, Chattopadhyay S, Mukherjee S K. Attenuation of Pseudomonas aeruginosa quorum sensing, virulence and biofilm formation by extracts of Andrographis paniculata. Microb Pathog, 2017; 113: 85-93.

21. Bottomley M J, Muraglia E, Bazzo R, Carfi A. Molecular insights into quorum sensing in the human pathogen Pseudomonas aeruginosa from the structure of the virulence regulator LasR bound to its autoinducer. J Biol Chem, 2007; 282: 13592-13600.

22. Hussain F M, Ahmad I, Baig M H, Khan M S, Khan M S, Hassan I, Al-Shabib N A. Broad-spectrum inhibition of $\mathrm{AHL}$-regulated virulence factors and biofilms by sub-inhibitory concentrations of ceftazidime. RSC Adv, 2016; 6: 27952-27962.

23. Maroyi A. Traditional use of medicinal plants in south-central Zimbabwe: review and perspectives. J Ethnobiol Ethnomed, 2013; 9: 31.

24. Pandey M M, Rastogi S, Rawat A K S. Indian traditional ayurvedic system of medicine and nutritional supplementation. Evid base Compl Alternative Med, 2013; 2013: Article ID 376327.

25. Vasavi H S, Arun A B, Rekha P D. Inhibition of quorum sensing in Chromobacterium violaceum by Syzygium cumini L. and Pimenta dioica L. Asian Pac J Trop Biomed, 2013; 3(12): 954-959.

26. Chong Y M, Yin W F, Ho C Y, Mustafa M R, Hadi H A, Awang K, Narrima P, Koh C L, Appleton D $\mathrm{R}$, Chan K G. Malabaricone C from Myristica cinnamomea exhibits anti-quorum sensing activity. J Nat Prod, 2011; 74(10): 2261-2264.

27. Pejin B, Ciric A, Glamoclija J, Nikolic M, Sokovic M. In vitro anti-quorum sensing activity of phytol. Nat Prod Res, 2015; 29(4): 374-377.

28. Darch S E, McNally A, Harrison F, Corander J, Barr H L, Paszkiewicz K, Holden S, Fogarty A, Crusz S A, Diggle S P. Recombination is a key driver of genomic and phenotypic diversity in a Pseudomonas aeruginosa population during cystic fibrosis infection. Sci Rep, 2015; 5: 7649.

29. Frederiksen R F, Paspaliari D K, Larsen T, Storgaard B G, Larsen M H, Ingmer H, Palcic M M, Leisner J J. Bacterial chitinases and chitin-binding proteins as virulence factors. Microbiol, 2013; 
159: 833-847.

30. Gholami S, Tabatabaei M, Sohrabi N. Comparison of biofilm formation and antibiotic resistance pattern of Pseudomonas aeruginosa in human and environmental isolates. Microb Pathog, 2017; 109: 94-98.

31. Issac Abraham S V, Palani A, Ramaswamy B R, Shunmugiah K P, Arumugam V R. Antiquorum sensing and antibiofilm potential of Capparis spinosa. Arch Med Res, 2011; 42(8): 658-668.

32. Periasamy S, Nair H A S, Lee K W K, Ong J, Goh J Q J, Kjelleberg S, Rice S A. Pseudomonas aeruginosa PAO1 exopolysaccharides are important for mixed species biofilm community development and stress tolerance. Front Microbiol, 2015; 6: 851.

33. Nickzad A, Deziel E. 2013. The involvement of rhamnolipids in microbial cell adhesion and biofilm development - an approach for control?. Lett Appl Microbiol, 2013; 58: 447-453.

34. Al-Haidari R A, Shaaban M I, Ibrahim S, Mohamed G A. Anti-quorum sensing activity of some medicinal plants. African J Trad Compl Alternative Med, 2016; 13(5): 67-71. 